\title{
Assessment of Cephalic and Facial Indices: A proof for Ethnic and Sexual Dimorphism
}

\author{
Twisha Shah ${ }^{1}$, Manish B Thaker ${ }^{2}$ and Shobhana K Menon ${ }^{{ }^{1}}$ \\ ${ }^{1}$ Department of Forensic Science, Gujarat University, Ahmedabad, Gujarat, India \\ ${ }^{2}$ Associate Professor Statistics Department, M.G Science Institute, Ahmedabad, Gujarat, India
}

*Corresponding author: Shobhana K Menon, Department of Forensic Science, Gujarat University, Ahmedabad 380009, Gujarat, India, Tel: +91-079-26300435, E-mail: shobhanamenon07@gmail.com

Citation: Twisha Shah, Manish B Thaker, Shobhana K Menon (2015) Assessment of Cephalic and Facial Indices: A proof for Ethnic and Sexual Dimorphism. J Forensic Sci Criminol 3(1): 101. doi: 10.15744/23489804.2.401

Received Date: January 13, 2015 Accepted Date: February 25, 2015 Published Date: February 27, 2015

\begin{abstract}
Cephalofacial measurements and indices are used to estimate sex and race. The aim of this is to document the craniofacial characteristics and sex differences from cephalic index and facial index of the studied groups. Maximum head length, maximum head width, morphological facial length and morphological facial width were determined for 901 Gujarati (676 male, 225 female) and 300 Non-Gujarati (100 male, 100 female) of Gujarat, India. The standard deviation of cephalic and facial index of Gujarati is 5.3345 and 6.0040 and of Non-Gujarati is 7.3472 and 6.0525 respectively. The mean cephalic index and facial index in study were 77.20 and 75.19 respectively in Gujarati and 74.13 and 75.17 respectively in Non-Gujarati. According to the cephalic index Gujarati subjects (40.2\% males, $42.7 \%$ females) mainly belongs to mesocephalic and dolicocephalic and Non-Gujarati subjects belongs to dolicocephalic ( $80 \%$ males) and brachycephalic (30\% females). According to the total facial index both the studied groups are mainly distributed into hypereuryprosopic followed by euryprosopic types of the face.
\end{abstract}

Keywords: Forensic Science; Anthropometry; Cephalic index; Facial index; Gujarat

\section{Introduction}

India is a nation with such a complex and varied ethnic composition of its population. No two people can ever be alike in all their measureable traits. Indian population is classified on the basis of anthropometric data collected from various population of India. Human face curve has always been an interesting topic for anatomists, anthropologists, plastic surgeons and artists. Physical anthropologists have been measuring skull for years and obtained results enabled them to trace the relationship between the races as they believe that the form of skull remain the same in each race and only different races show different facial index and cephalic index [1]. Cephalofacial measurement plays a very important role in personal identification in forensic application.

Cephalometry technique summarizes the anatomical complexes of the head and face of human being living within a similar geography. Feature measurements provide a system for metrical recording of size and proportions of craniofacial features instead of subjective impressions. These recordings yield a numerical expression which is important in evaluating population by comparison of head form and face form.

For evaluation of variation in cephalic index and facial index, standard charts should be followed. Many studies have been conducted to apply techniques of anthropometry to study the dimensions of body and its proportions based on age, sex, geographical and genetic factors. Many reported works have been published on cephalic index of Gujarati population [2-4]. These works were done on comparatively smaller age group and somewhat lesser samples. Also, very few published work has yet been reported on facial index of Gujarati population. Earlier reported works on facial indices were measured using CT scan images [5] and photographs [6]. The need for study of morphological facial measurements of Gujarati group has not come under much attention so far. Hence driven by the need this work necessitates the study of head and face type of Gujarati people and comparing the results with Non-Gujarati samples. Geometrical variation not only helps to understand the variation in the body measurement in variety of population but also helps to make a databank useful for computer based technologies. This anthropometric data of the selected population represents the data on the cephalofacial anthropometry. Ethnic differences are analyzed using facial and cephalic indices between the studied groups to formulate standards to that particular population. The values of these indices vary among different ethnicities which are useful for morphometric investigation. Indices express the ratio of landmarks of one distance to another distance of an individual. Cephalic index is the measurement related to the shape of the skull. Head length is the distance from glabella to opisthocranion and head width is the distance between two euryons. According to Williams et al [7] the cephalic index were calculated as: 


$$
\text { Cephalic Index }=\frac{\text { Maximum Head Width }(\text { eu-eu }) \times 100}{\text { Maximum Head Length }(g-o p)}
$$

And Facial index is the relation of the face to its maximum width between two zygomatic points to the height of the face from nasion to gnathion. Facial index was determined by Williams et al. [7] and was calculated using the following formula: Facial Index $=$ Morphological Facial Length (n-gn) $\times 100$ Morphological Facial Width (zy-zy). Hence the cephalic and facial indices can be very important evidence towards the identity of the unknown person. Such data can be useful in forensic science, during surgeries and for comparative study.

\section{Materials and Methods}

The present work was undertaken to determine cephalic index and facial index particularly in relation to male and female and to study the distribution of the common head and face types in Gujarati and Non-Gujarati population of Gujarat. The cross-sectional study was conducted on 901 Gujarati $(676=$ male and $225=$ female $)$ and 300 Non-Gujarati $(200=$ male and $100=$ female $)$ who reside in the Ahmedabad district of Gujarat, India. Totally 1201 adult volunteers were studied whose age range between 20-50 years. Subjects showing any type of cephalofacial deformity were excluded from the study. Before starting study, the aims and objectives of the study was made understand to the volunteers and informed consent of the subjects was taken. Subjects whose forefather originates from Gujarat and born and bought up in Gujarat state were considered as Gujarati. Subject who resides in Gujarat but his ancestral background and origin is not Gujarat was regarded as Non-Gujarati. A set of questionnaire comprising the demographic profile, ancestral background and other necessary information were verified before considering them under Gujarati and Non-Gujarati. Non-Gujarati subjects include people from various ethnic groups viz. Bengali, Marathi, Bihari, Odissi, Punjabi, Rajasthani, Nepali, South Indian, etc. Measurements on the subjects were taken by locating the landmarks properly on the face.

All the landmarks were palpated and marked lightly with pen before taking the measurements. The head of the subject were allowed to rest in eye-ear plane or Frank-furt plane i.e., tragion and right orbitale must lie in the same plane. Subjects were made to sit on low raise stool and should not change his/her position while taking measurements. All the measurements were taken thrice and mean was calculated to ensure accuracy of the measurement taken. All the measurements have been taken following the techniques of Martin and Saller (1957) [8] and Singh and Bhasin (1989) [9]. Martin recommends that for head measurements an error of 0.5 to $1.0 \mathrm{~mm}$; for head height $2.0 \mathrm{~mm}$; for most of the body measurements 3.0 to $5.0 \mathrm{~mm}$ and for stature and span 10.0 $\mathrm{mm}$ may be allowed [9]. Definations of landmarks, measuring techniques and instrument used are described according to Indera P Singh \& M.K. Bhasin [9]. All measurements are recorded to the nearest millimetre or centimetre. The anatomical landmarks are defined as follows:-

I. GLABELLA (g): A point above the nasal root between the eyebrows and intersected by mid-saggital plane.

II. OPISTHOCRANION (op): It is the most posterior point on the posterior protuberance of the head in the mid sagittal plane. This point is determined by measuring maximum head length.

III. EURYON (eu): It is the most laterally placed point on the sides of the head. This point can be determined by measuring the maximum head breadth.

IV. GNATHION (gn): It is the lowest point on the lower margin of the lower jaw intersected by the mid-sagittal plane. This point can be palpated on the lower jaw from behind and slightly anterior to chin.

V. NASION (n): It is the point on the nasal root intersected by mid sagittal plane. Nasal root is not the depression of the nose but at the nasofrontal suture which can be felt by slightly probing the root of the nose. Note that nasion usually lies in the level of the medial end of the eye brows mostly at the lower margins and not at the height of the eye brows.

VI. ZYGION (zy): It is the most lateral placed point on the zygomatic arch. These points are determined by taking bizygomatic breadth.

The maximum head length was measured with the help of spreading calliper from glabella to opisthocranion. Maximum head width was measured as the maximum transverse diameter between euryon to euryon using spreading calliper. Morphological facial length (distance from nasion to gnathion) and facial width (distance between two zygomatic landmarks) was measured using sliding caliper.

All the data were analysed using Microsoft Excel and SPSS (Statistical Package for Social Sciences) version 22.0. Mean and Standard deviation were calculated. From the observations of the present study the parametric data were analyzed using independent sample t-test. Sample observations are independent (since the study is related to two groups only, hence ANOVA cannot be applied). Since the sample size is large it is assumed to follow normality. In an unpaired means the sample observations are taken from two different individuals hence the sample size is not necessarily equal.

$$
t=\frac{\bar{X}=\bar{Y}}{\mathrm{~s} \sqrt{\frac{1}{n_{1}}+\frac{1}{n_{2}}}} \text { followst distribution with }\left(n_{1}+n_{2}-2\right) d f \text {. }
$$

Where 


$$
\begin{aligned}
& S^{2}=\frac{\sum(X-\bar{X})^{2}+\sum\left(Y-\bar{Y}^{2}\right.}{\left(n_{1}+n_{2}-2\right)} \\
& \mathrm{X}=\text { Gujarati } \\
& \mathrm{Y}=\text { Non-Gujarati } \\
& \mathrm{N}_{1}=\text { Number of Gujarati } \\
& \mathrm{N}_{2}=\text { Number of Non-Gujarati }
\end{aligned}
$$

The analysis is carried out for the following hypothesis:

Hypothesis 1 : To test significant difference in the mean values of head length (Head width, facial length, facial width, cephalic index and facial index) for male (female) of Gujarati (Non- Gujarati).

$\mathrm{H}_{\mathrm{o}}: \mu_{\mathrm{m}}=\mu_{\mathrm{f}}$ or

$\mathrm{H}_{\mathrm{o}}: \mu_{\mathrm{m}} \neq \mu_{\mathrm{f}}$

where, $\mu=$ head length, head width, facial length, facial width, cephalic index and facial index

$$
\begin{aligned}
& \mu_{\mathrm{m}}=\text { Male } \\
& \mu_{\mathrm{f}}=\text { Female }
\end{aligned}
$$

Hypothesis 2: To test significant difference in the mean values of head length (Head width, facial length, facial width, cephalic index and facial index) for Gujarati (Non-Gujarati)

$$
\begin{aligned}
& \mathrm{H}_{\mathrm{o}}: \mu_{\mathrm{ng}}=\mu_{\mathrm{g}} \text { or } \\
& \mathrm{H}_{\mathrm{o}}: \mu_{\mathrm{ng}} \neq \mu_{\mathrm{g}}
\end{aligned}
$$

where, $\mu=$ head length, head width, facial length, facial width, cephalic index and facial index

$$
\begin{aligned}
& \mu_{n g}=\text { Non-Gujarati } \\
& \mu_{\mathrm{g}}=\text { Gujarati }
\end{aligned}
$$

The cephalic index and facial index types of head and face forms were classified as given by Martin and Saller (1957) [8] (Table 1 and 2).

\begin{tabular}{|l|l|l|}
\hline \multirow{2}{*}{ Head type } & \multicolumn{2}{|l|}{ Range of Index } \\
\cline { 2 - 3 } & Male & Female \\
\hline Dolichocephalic & $71.0-75.9$ & $72.0-76.9$ \\
\hline Mesocephalic & $76.0-80.9$ & $77.0-81.9$ \\
\hline Brachycephalic & $81.0-85.9$ & $82.0-86.4$ \\
\hline Hyperbrachycephalic & $86.0-90.9$ & $86.5-91.9$ \\
\hline Ultrabrachycephalic & $91.0-\mathrm{x}$ & $92.0-\mathrm{x}$ \\
\hline
\end{tabular}

Table 1: Classification of Head types according to Martin and Saller (1957) [8]

\begin{tabular}{|l|l|l|}
\hline \multirow{2}{*}{ Facial type } & \multicolumn{2}{|l|}{ Range of Index } \\
\cline { 2 - 3 } & Male & Female \\
\hline Hypereuryprosopic & $\mathrm{x}-78.9$ & $\mathrm{x}-76.9$ \\
\hline Euryprosopic & $79.0-83.9$ & $77.0-80.9$ \\
\hline Mesoprosopic & $84.0-87.9$ & $81.0-84.9$ \\
\hline Leptoprosopic & $88.0-92.9$ & $85.0-89.9$ \\
\hline Hyperleptoprosopic & $93.0-\mathrm{x}$ & $90.0-\mathrm{x}$ \\
\hline
\end{tabular}

Table 2: Classification of Facial types according to Martin and Saller (1957) [8]

\section{Results}

The findings of basic statistical parameters for the measured variables in treated subjects of Gujarati and Non-Gujarati were performed. N, mean, minimum, maximum, standard deviation and t-test of head height, head width, facial height, facial width, cephalic index and facial index of Gujarati and Non-Gujarati population are presented in Table 3 and 4 . The mean cephalic and facial index were compared between male and female subjects within the selected group of people (Figure 1 and 2). 


\begin{tabular}{|c|c|c|c|c|c|c|c|c|c|}
\hline Test Variable & Sex & $\mathbf{N}$ & Mini & Maxi & Mean & Std. Deviation & Std. Error Mean & $\mathbf{t}$ & Sig. (2- tailed) \\
\hline \multirow{2}{*}{$\begin{array}{l}\text { Maximum } \\
\text { Head Length }\end{array}$} & Male & 200 & 13.8 & 21.2 & $16.955^{*}$ & 1.6653 & .1178 & \multirow[b]{2}{*}{-4.164} & \multirow[b]{2}{*}{0.00} \\
\hline & Female & 100 & 14.5 & 19.3 & $17.738^{\star}$ & 1.2370 & .1237 & & \\
\hline \multirow{2}{*}{$\begin{array}{l}\text { Maximum } \\
\text { Head Width }\end{array}$} & Male & 200 & 9.6 & 15.9 & $11.974^{*}$ & 1.4985 & .1060 & \multirow{2}{*}{-13.811} & \multirow{2}{*}{0.00} \\
\hline & Female & 100 & 11.2 & 17.1 & $14.377^{\star}$ & 1.2494 & .1249 & & \\
\hline \multirow{2}{*}{$\begin{array}{l}\text { Morphological } \\
\text { Facial Width }\end{array}$} & Male & 200 & 9.6 & 14.6 & $11.6130^{*}$ & 1.35277 & .09566 & \multirow{2}{*}{-12.365} & \multirow{2}{*}{0.00} \\
\hline & Female & 100 & 10.8 & 15.1 & $13.4960^{*}$ & .98739 & .09874 & & \\
\hline \multirow{2}{*}{$\begin{array}{l}\text { Morphological } \\
\text { Facial Length }\end{array}$} & Male & 200 & 6 & 12.2 & $8.653^{*}$ & 1.4542 & .1028 & & \multirow{2}{*}{0.00} \\
\hline & Female & 100 & 7.2 & 11.8 & $10.389^{*}$ & .9831 & .0983 & & \\
\hline \multirow{2}{*}{ Cephalic index } & Male & 200 & 56.7 & 81.5 & $70.615^{*}$ & 5.0987 & .3605 & \multirow{2}{*}{-15.93} & \multirow{2}{*}{0.00} \\
\hline & Female & 100 & 70.0 & 89.9 & $81.170^{*}$ & 5.9793 & .5979 & & \\
\hline \multirow{2}{*}{ Facial index } & Male & 200 & 60.5 & 91.0 & $74.283^{*}$ & 6.4919 & .4590 & \multirow{2}{*}{-3.67} & \multirow{2}{*}{0.00} \\
\hline & Female & 100 & 66.4 & 84.3 & $76.952^{*}$ & 4.5965 & .4597 & & \\
\hline
\end{tabular}

${ }^{*}$-value $<0.05$

Table 3: Descriptive Statistics of Non-Gujarati $(\mathrm{n}=300)$

\begin{tabular}{|c|c|c|c|c|c|c|c|c|c|}
\hline Test Variable & Sex & $\mathbf{N}$ & Mini & Maxi & Mean & Std. Deviation & Std. Error Mean & $\mathbf{t}$ & Sig. (2- tailed) \\
\hline \multirow{2}{*}{$\begin{array}{l}\text { Maximum } \\
\text { Head Length }\end{array}$} & Male & 676 & 14.0 & 21.4 & $17.790^{*}$ & 1.5218 & .0585 & \multirow{2}{*}{14.967} & \multirow{2}{*}{0.00} \\
\hline & Female & 225 & 13.6 & 18.8 & $16.012^{*}$ & 1.6087 & .1072 & & \\
\hline \multirow{2}{*}{$\begin{array}{l}\text { Maximum } \\
\text { Head Width }\end{array}$} & Male & 676 & 10.1 & 19.0 & $13.729^{*}$ & 1.5211 & .0585 & \multirow{2}{*}{11.404} & \multirow{2}{*}{0.00} \\
\hline & Female & 225 & 9.6 & 18.6 & $12.388^{*}$ & 1.5467 & .1031 & & \\
\hline \multirow{2}{*}{$\begin{array}{l}\text { Morphological } \\
\text { Facial Width }\end{array}$} & Male & 676 & 9.8 & 16.8 & $13.0749^{*}$ & 1.54196 & .05931 & \multirow{2}{*}{14.25} & \multirow[b]{2}{*}{0.00} \\
\hline & Female & 225 & 9 & 14.5 & $11.4036^{*}$ & 1.46424 & .09762 & & \\
\hline \multirow{2}{*}{$\begin{array}{l}\text { Morphological } \\
\text { Facial Length }\end{array}$} & Male & 676 & 6.3 & 12.6 & $9.853^{\star}$ & 1.3680 & .0526 & \multirow{2}{*}{12.219} & \multirow{2}{*}{0.00} \\
\hline & Female & 225 & 6.1 & 12 & $8.542^{*}$ & 1.4680 & .0979 & & \\
\hline \multirow{2}{*}{ Cephalic index } & Male & 676 & 61.4 & 103.3 & 77.149 & 5.2318 & .2012 & \multirow{2}{*}{0.566} & \multirow{2}{*}{0.571} \\
\hline & Female & 225 & 60.8 & 103.3 & 77.381 & 5.6404 & .3760 & & \\
\hline \multirow[t]{2}{*}{ Facial index } & Male & 676 & 57.3 & 92.2 & 75.354 & 5.7497 & .2211 & \multirow{2}{*}{1.348} & \multirow{2}{*}{0.178} \\
\hline & Female & 225 & 54.0 & 94.5 & 74.731 & 6.7026 & .4468 & & \\
\hline
\end{tabular}

${ }^{*}$ p-value $<0.05$

Table 4: Descriptive Statistics of Gujarati $(\mathrm{n}=901)$

The mean values and t-test of all the test variables were performed to test the existence of any significant difference. From the collected data, statistics were analyzed and results are presented in tabular form (Table 5).

\begin{tabular}{|c|c|c|c|c|c|c|c|}
\hline Test Variable & Native & $\mathbf{N}$ & Mean & Std. Deviation & Std. Error Mean & $\mathrm{t}$ & Sig. (2- tailed) \\
\hline \multirow{2}{*}{$\begin{array}{l}\text { Maximum } \\
\text { Head Length }\end{array}$} & Non Gujarati & 300 & 17.216 & 1.5777 & .0911 & \multirow{2}{*}{-1.157} & \multirow{2}{*}{.247} \\
\hline & Gujarati & 901 & 17.346 & 1.7246 & .0575 & & \\
\hline \multirow{2}{*}{$\begin{array}{l}\text { Maximum } \\
\text { Head Width }\end{array}$} & Non Gujarati & 300 & $12.775^{*}$ & 1.8163 & .1049 & \multirow{2}{*}{-5.524} & \multirow{2}{*}{.000} \\
\hline & Gujarati & 901 & $13.394^{*}$ & 1.6333 & .0544 & & \\
\hline \multirow{2}{*}{$\begin{array}{l}\text { Morphological } \\
\text { Facial Width }\end{array}$} & Non Gujarati & 300 & $12.2407^{*}$ & 1.52687 & .08815 & \multirow{2}{*}{-3.796} & \multirow{2}{*}{.000} \\
\hline & Gujarati & 901 & $12.6575^{*}$ & 1.68546 & .05615 & & \\
\hline \multirow{2}{*}{$\begin{array}{l}\text { Morphological } \\
\text { Facial Length }\end{array}$} & Non Gujarati & 300 & $9.232^{*}$ & 1.5490 & .0894 & \multirow{2}{*}{-2.909} & \multirow{2}{*}{.004} \\
\hline & Gujarati & 901 & $9.526^{*}$ & 1.5041 & .0501 & & \\
\hline \multirow{2}{*}{$\begin{array}{l}\text { Cephalic } \\
\text { index }\end{array}$} & Non Gujarati & 300 & $74.133^{*}$ & 7.3472 & .4242 & \multirow{2}{*}{-7.814} & \multirow{2}{*}{.000} \\
\hline & Gujarati & 901 & $77.207^{*}$ & 5.3345 & .1777 & & \\
\hline \multirow{2}{*}{ Facial index } & Non Gujarati & 300 & 75.173 & 6.0525 & .3494 & \multirow{2}{*}{-.065} & \multirow{2}{*}{.948} \\
\hline & Gujarati & 901 & 75.199 & 6.0040 & .2000 & & \\
\hline
\end{tabular}

${ }^{*} \mathrm{p}$-value $<0.05$

Table 5: t-test of all variables $(n=1201)$ 
Addition, the frequency of the most common head and facial types found from the values of cephalic and facial indices were studied in the treated subjects (Table 6 and 7 ).

\begin{tabular}{|c|c|c|c|c|c|c|c|}
\hline \multirow{2}{*}{\multicolumn{2}{|c|}{ Cephalo Types }} & \multicolumn{2}{|c|}{ Male } & \multicolumn{2}{|c|}{ Female } & \multicolumn{2}{|c|}{ Both } \\
\hline & & Frequency & Percent & Frequency & Percent & Frequency & Percent \\
\hline \multirow{5}{*}{ 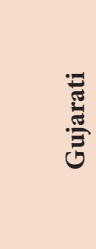 } & Dolichocephalic & 160 & $80.0 \%$ & 27 & $27.0 \%$ & 187 & $62.3 \%$ \\
\hline & Mesocephalic & 36 & $18.0 \%$ & 20 & $20.0 \%$ & 56 & $18.7 \%$ \\
\hline & Brachycephalic & 4 & $2.0 \%$ & 30 & $30.0 \%$ & 34 & $11.3 \%$ \\
\hline & Hyperbrachycephalic & 0 & $0.0 \%$ & 23 & $23.0 \%$ & 23 & $7.7 \%$ \\
\hline & Total & 200 & $100 \%$ & 100 & $100 \%$ & 300 & $100 \%$ \\
\hline \multicolumn{2}{|c|}{ Facial Types } & Frequency & Percent & Frequency & Percent & Frequency & Percent \\
\hline \multirow{5}{*}{ 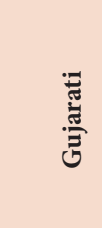 } & Hypereuryprosopic & 152 & $76.0 \%$ & 37 & $37.0 \%$ & 189 & $63.0 \%$ \\
\hline & Euryprosopic & 30 & $15.0 \%$ & 45 & $45.0 \%$ & 75 & $25.0 \%$ \\
\hline & Mesoprosopic & 14 & $7.0 \%$ & 18 & $18.0 \%$ & 32 & $10.7 \%$ \\
\hline & Leptoprosopic & 4 & $2.0 \%$ & 0 & $0.0 \%$ & 4 & $1.3 \%$ \\
\hline & Total & 200 & $100 \%$ & 100 & $100 \%$ & 300 & $100 \%$ \\
\hline
\end{tabular}

Table 6: Frequency of Head types and Facial types of Non-Gujarati $(n=300)$

\begin{tabular}{|c|c|c|c|c|c|c|c|}
\hline \multirow{2}{*}{\multicolumn{2}{|c|}{ Cephalo Types }} & \multicolumn{2}{|c|}{ Male } & \multicolumn{2}{|c|}{ Female } & \multicolumn{2}{|c|}{ Both } \\
\hline & & \multirow{2}{*}{$\begin{array}{l}\text { Frequency } \\
269 \\
\end{array}$} & \multirow{2}{*}{\begin{tabular}{|l|} 
Percent \\
$39.8 \%$ \\
\end{tabular}} & \multirow{2}{*}{$\begin{array}{l}\text { Frequency } \\
96\end{array}$} & \multirow{2}{*}{$\begin{array}{l}\text { Percent } \\
42.7 \%\end{array}$} & \multirow{2}{*}{$\begin{array}{l}\text { Frequency } \\
365\end{array}$} & \multirow{2}{*}{$\begin{array}{l}\text { Percent } \\
40.5 \% \\
\end{array}$} \\
\hline \multirow{6}{*}{ 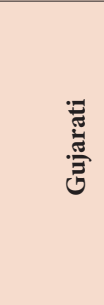 } & Dolichocephalic & & & & & & \\
\hline & Mesocephalic & 272 & $40.2 \%$ & 95 & $42.2 \%$ & 367 & $40.7 \%$ \\
\hline & Brachycephalic & 105 & $15.5 \%$ & 23 & $10.2 \%$ & 128 & $14.2 \%$ \\
\hline & Hyperbrachycephalic & 24 & $3.6 \%$ & 9 & $4.0 \%$ & 33 & $3.7 \%$ \\
\hline & Ultrabrachycephalic & 6 & $0.9 \%$ & 2 & $0.9 \%$ & 8 & $0.9 \%$ \\
\hline & Total & 676 & $100 \%$ & 225 & $100 \%$ & 901 & $100 \%$ \\
\hline \multicolumn{2}{|c|}{ Facial Types } & Frequency & Percent & Frequency & Percent & Frequency & Percent \\
\hline \multirow{6}{*}{ } & Hypereuryprosopic & 514 & $76.0 \%$ & 135 & $60.0 \%$ & 649 & $72.0 \%$ \\
\hline & Euryprosopic & 111 & $16.4 \%$ & 53 & $23.6 \%$ & 164 & $18.2 \%$ \\
\hline & Mesoprosopic & 36 & $5.3 \%$ & 27 & $12.0 \%$ & 63 & $7.0 \%$ \\
\hline & Leptoprosopic & 15 & $2.2 \%$ & 6 & $2.7 \%$ & 21 & $2.3 \%$ \\
\hline & Hyperleptoprosopic & 0 & $0.0 \%$ & 4 & $1.8 \%$ & 4 & $0.4 \%$ \\
\hline & Total & 676 & $100 \%$ & 225 & $100 \%$ & 901 & $100 \%$ \\
\hline
\end{tabular}

Table 7: Frequency of Head types and Facial types of Gujarati ( $n=901)$

The relationship between head types and facial types were known by performing cross-tab (Table 8 and 9)

\begin{tabular}{|c|c|c|c|c|c|c|c|}
\hline & & & \multicolumn{4}{|c|}{ Face type } & \multirow[b]{2}{*}{ Total } \\
\hline & \multicolumn{2}{|c|}{ Sex } & $\begin{array}{c}\text { Hypere- } \\
\text { urypros } \\
\text { opic }\end{array}$ & $\begin{array}{c}\text { Euryproso } \\
\text { pic }\end{array}$ & $\begin{array}{l}\text { Mesopro- } \\
\text { so pic }\end{array}$ & $\begin{array}{c}\text { Leptoproso } \\
\text { pic }\end{array}$ & \\
\hline \multirow{8}{*}{$\frac{\stackrel{\nu}{\Sigma}}{\Sigma}$} & \multirow{6}{*}{ 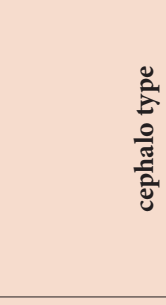 } & \multirow{2}{*}{ Dolichocephalic } & 120 & 24 & 12 & 4 & 160 \\
\hline & & & $60.0 \%$ & $12.0 \%$ & $6.0 \%$ & $2.0 \%$ & $80.0 \%$ \\
\hline & & \multirow{2}{*}{ Mesocephalic } & 32 & 4 & 0 & 0 & 36 \\
\hline & & & $16.0 \%$ & $2.0 \%$ & $0.0 \%$ & $0.0 \%$ & $18.0 \%$ \\
\hline & & \multirow{2}{*}{ Brachycephalic } & 0 & 2 & 2 & 0 & 4 \\
\hline & & & $0.0 \%$ & $1.0 \%$ & $1.0 \%$ & $0.0 \%$ & $2.0 \%$ \\
\hline & \multirow{2}{*}{\multicolumn{2}{|c|}{ Total }} & 152 & 30 & 14 & 4 & 200 \\
\hline & & & $76.0 \%$ & $15.0 \%$ & $7.0 \%$ & $2.0 \%$ & $100.0 \%$ \\
\hline
\end{tabular}




\begin{tabular}{|c|c|c|c|c|c|c|c|}
\hline \multirow{2}{*}{\multicolumn{3}{|c|}{ Sex }} & \multicolumn{4}{|c|}{ Face type } & \multirow{3}{*}{$\begin{array}{l}\text { Total } \\
27 \\
\end{array}$} \\
\hline & & & \multirow{2}{*}{$\begin{array}{l}\text { Hypereurypros opic } \\
13\end{array}$} & \multirow{2}{*}{$\begin{array}{l}\text { Euryproso pic } \\
9\end{array}$} & \multirow{2}{*}{$\begin{array}{l}\text { Mesoproso pic } \\
5\end{array}$} & \multirow{2}{*}{\begin{tabular}{|l} 
Leptoproso pic \\
0 \\
\end{tabular}} & \\
\hline \multirow{10}{*}{ 噉 } & \multirow{8}{*}{ 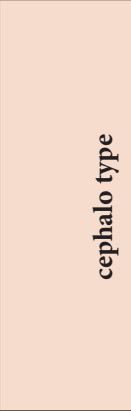 } & \multirow{2}{*}{ Dolichocephalic } & & & & & \\
\hline & & & $13.0 \%$ & $9.0 \%$ & $5.0 \%$ & 0 & 27.0 \\
\hline & & \multirow{2}{*}{ Mesocephalic } & 10 & 5 & 5 & 0 & 20 \\
\hline & & & $10.0 \%$ & $5.0 \%$ & $5.0 \%$ & 0 & 20.0 \\
\hline & & \multirow{2}{*}{ Brachycephalic } & 6 & 18 & 6 & 0 & 30 \\
\hline & & & $6.0 \%$ & $18.0 \%$ & $6.0 \%$ & 0 & $30.0 \%$ \\
\hline & & \multirow{2}{*}{ Hyperbrachycep halic } & 8 & 13 & 2 & 0 & 23 \\
\hline & & & $8.0 \%$ & $13.0 \%$ & $2.0 \%$ & 0 & $23.0 \%$ \\
\hline & \multirow{2}{*}{\multicolumn{2}{|c|}{ Total }} & 37 & 45 & 18 & 0 & 100 \\
\hline & & & $37.0 \%$ & $45.0 \%$ & $18.0 \%$ & 0 & $100.0 \%$ \\
\hline \multirow{10}{*}{ } & \multirow{8}{*}{ 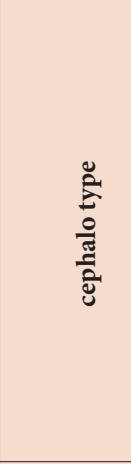 } & \multirow{2}{*}{ Dolichocepzalic } & 133 & 33 & 17 & 4 & 187 \\
\hline & & & $44.3 \%$ & $11.0 \%$ & $5.7 \%$ & $1.3 \%$ & $62.3 \%$ \\
\hline & & \multirow{2}{*}{ Mesocephalic } & 42 & 9 & 5 & 0 & 56 \\
\hline & & & $14.0 \%$ & $3.0 \%$ & $1.7 \%$ & $0.0 \%$ & $18.7 \%$ \\
\hline & & \multirow{2}{*}{ Brachycephalic } & 6 & 20 & 8 & 0 & 34 \\
\hline & & & $2.0 \%$ & $6.7 \%$ & $2.7 \%$ & $0.0 \%$ & $11.3 \%$ \\
\hline & & \multirow{2}{*}{ Hyperbrachycep halic } & 8 & 13 & 2 & 0 & 23 \\
\hline & & & $2.7 \%$ & $4.3 \%$ & $0.7 \%$ & $0.0 \%$ & $7.7 \%$ \\
\hline & \multirow{2}{*}{\multicolumn{2}{|c|}{ Total }} & 189 & 75 & 32 & 4 & 300 \\
\hline & & & $63.0 \%$ & $25.0 \%$ & $10.7 \%$ & $1.3 \%$ & 100.0 \\
\hline
\end{tabular}

Table 8: Facial Index and Cranial Index Cross Tab of Non-Gujarati $(n=300)$

\begin{tabular}{|c|c|c|c|c|c|c|c|c|}
\hline & & \multirow{2}{*}{ Sex } & \multicolumn{4}{|c|}{ Face type } & \multirow[b]{2}{*}{ Hyperleptoprosopic } & \multirow{2}{*}{ Total } \\
\hline & & & Hypereuryprosopic & Euryprosopic & Mesoprosopic & Leptoprosopic & & \\
\hline \multirow{12}{*}{$\stackrel{\pi}{\Sigma}^{\frac{\pi}{\Sigma}}$} & \multirow{10}{*}{ 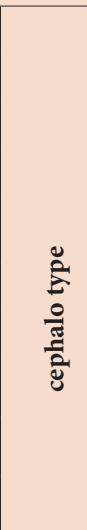 } & \multirow{2}{*}{ Dolichocephalic } & 184 & 55 & 22 & 8 & 0 & 269 \\
\hline & & & $27.2 \%$ & $8.1 \%$ & $3.3 \%$ & $1.2 \%$ & 0 & $39.8 \%$ \\
\hline & & \multirow{2}{*}{ Mesocephalic } & 208 & 46 & 11 & 7 & 0 & 272 \\
\hline & & & $30.8 \%$ & $6.8 \%$ & $1.6 \%$ & $1.0 \%$ & 0 & $40.2 \%$ \\
\hline & & \multirow{2}{*}{ Brachycephalic } & 93 & 9 & 3 & 0 & 0 & 105 \\
\hline & & & $13.8 \%$ & $1.3 \%$ & $0.4 \%$ & $0.0 \%$ & 0 & $15.5 \%$ \\
\hline & & \multirow{2}{*}{ Hyperbrachycephalic } & 24 & 0 & 0 & 0 & 0 & 24 \\
\hline & & & $3.6 \%$ & $0.0 \%$ & $0.0 \%$ & $0.0 \%$ & 0 & $3.6 \%$ \\
\hline & & \multirow{2}{*}{ Ultrabrachycephalic } & 5 & 1 & 0 & 0 & 0 & 6 \\
\hline & & & $0.7 \%$ & $0.1 \%$ & $0.0 \%$ & $0.0 \%$ & 0 & $0.9 \%$ \\
\hline & \multirow{2}{*}{\multicolumn{2}{|c|}{ Total }} & 514 & 111 & 36 & 15 & 0 & 676 \\
\hline & & & $76.0 \%$ & $16.4 \%$ & $5.3 \%$ & $2.2 \%$ & 0 & $100.0 \%$ \\
\hline \multirow{6}{*}{ 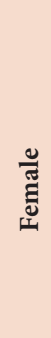 } & \multirow{6}{*}{ 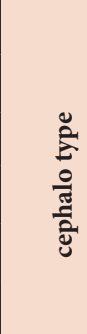 } & \multirow{2}{*}{ Dolichocephalic } & 56 & 21 & 9 & 6 & 4 & 96 \\
\hline & & & $24.9 \%$ & $9.3 \%$ & $4.0 \%$ & $2.7 \%$ & $1.8 \%$ & $42.7 \%$ \\
\hline & & \multirow{2}{*}{ Mesocephalic } & 55 & 26 & 14 & 0 & 0 & 95 \\
\hline & & & $24.4 \%$ & $11.6 \%$ & $6.2 \%$ & $0.0 \%$ & $0.0 \%$ & $42.2 \%$ \\
\hline & & \multirow{2}{*}{ Brachycephalic } & 15 & 4 & 4 & 0 & 0 & 23 \\
\hline & & & $6.7 \%$ & $1.8 \%$ & $1.8 \%$ & $0.0 \%$ & $0.0 \%$ & $10.2 \%$ \\
\hline
\end{tabular}




\begin{tabular}{|c|c|c|c|c|c|c|c|c|}
\hline & & \multirow{2}{*}{ Sex } & \multicolumn{4}{|c|}{ Face type } & \multirow[b]{2}{*}{ Hyperleptoprosopic } & \multirow{2}{*}{ Tota } \\
\hline & & & Hypereuryprosopic & Euryprosopic & Mesoprosopic & Leptoprosopic & & \\
\hline & & \multirow{2}{*}{ Hyperbrachycephalic } & 7 & 2 & 0 & 0 & 0 & 9 \\
\hline & & & $3.1 \%$ & $0.9 \%$ & $0.0 \%$ & $0.0 \%$ & $0.0 \%$ & $4.0 \%$ \\
\hline & & \multirow{2}{*}{ Ultrabrachycephalic } & 2 & 0 & 0 & 0 & 0 & 2 \\
\hline & & & $0.9 \%$ & $0.0 \%$ & $0.0 \%$ & $0.0 \%$ & $0.0 \%$ & $0.9 \%$ \\
\hline & \multirow{2}{*}{\multicolumn{2}{|c|}{ Total }} & 135 & 53 & 27 & 6 & 4 & 225 \\
\hline & & & $60.0 \%$ & $23.6 \%$ & $12.0 \%$ & $2.7 \%$ & $1.8 \%$ & $100.0 \%$ \\
\hline \multirow{12}{*}{ 量 } & \multirow{10}{*}{ 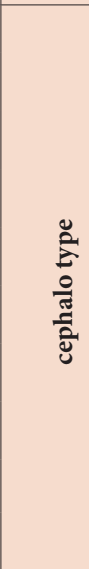 } & Dolichocephalic & 240 & 76 & 31 & 14 & 4 & 365 \\
\hline & & & $26.6 \%$ & $8.4 \%$ & $3.4 \%$ & $1.6 \%$ & $0.4 \%$ & $40.5 \%$ \\
\hline & & Mesocephalic & 263 & 72 & 25 & 7 & 0 & 367 \\
\hline & & & $29.2 \%$ & $8.0 \%$ & $2.8 \%$ & $0.8 \%$ & $0.0 \%$ & $40.7 \%$ \\
\hline & & Brachycephalic & 108 & 13 & 7 & 0 & 0 & 128 \\
\hline & & & $12.0 \%$ & $1.4 \%$ & $0.8 \%$ & $0.0 \%$ & $0.0 \%$ & $14.2 \%$ \\
\hline & & Hyperbrachycephalic & 31 & 2 & 0 & 0 & 0 & 33 \\
\hline & & & $3.4 \%$ & $0.2 \%$ & $0.0 \%$ & $0.0 \%$ & $0.0 \%$ & $3.7 \%$ \\
\hline & & Ultrabrachycephalic & 7 & 1 & 0 & 0 & 0 & 8 \\
\hline & & & $0.8 \%$ & $0.1 \%$ & $0.0 \%$ & $0.0 \%$ & $0.0 \%$ & $0.9 \%$ \\
\hline & \multirow{2}{*}{\multicolumn{2}{|c|}{ Total }} & 649 & 164 & 63 & 21 & 4 & 901 \\
\hline & & & $72.0 \%$ & $18.2 \%$ & $7.0 \%$ & $2.3 \%$ & $0.4 \%$ & $100.0 \%$ \\
\hline
\end{tabular}

Table 9: Facial Index and Cranial Index Cross Tab of Gujarati ( $\mathrm{n}=901)$

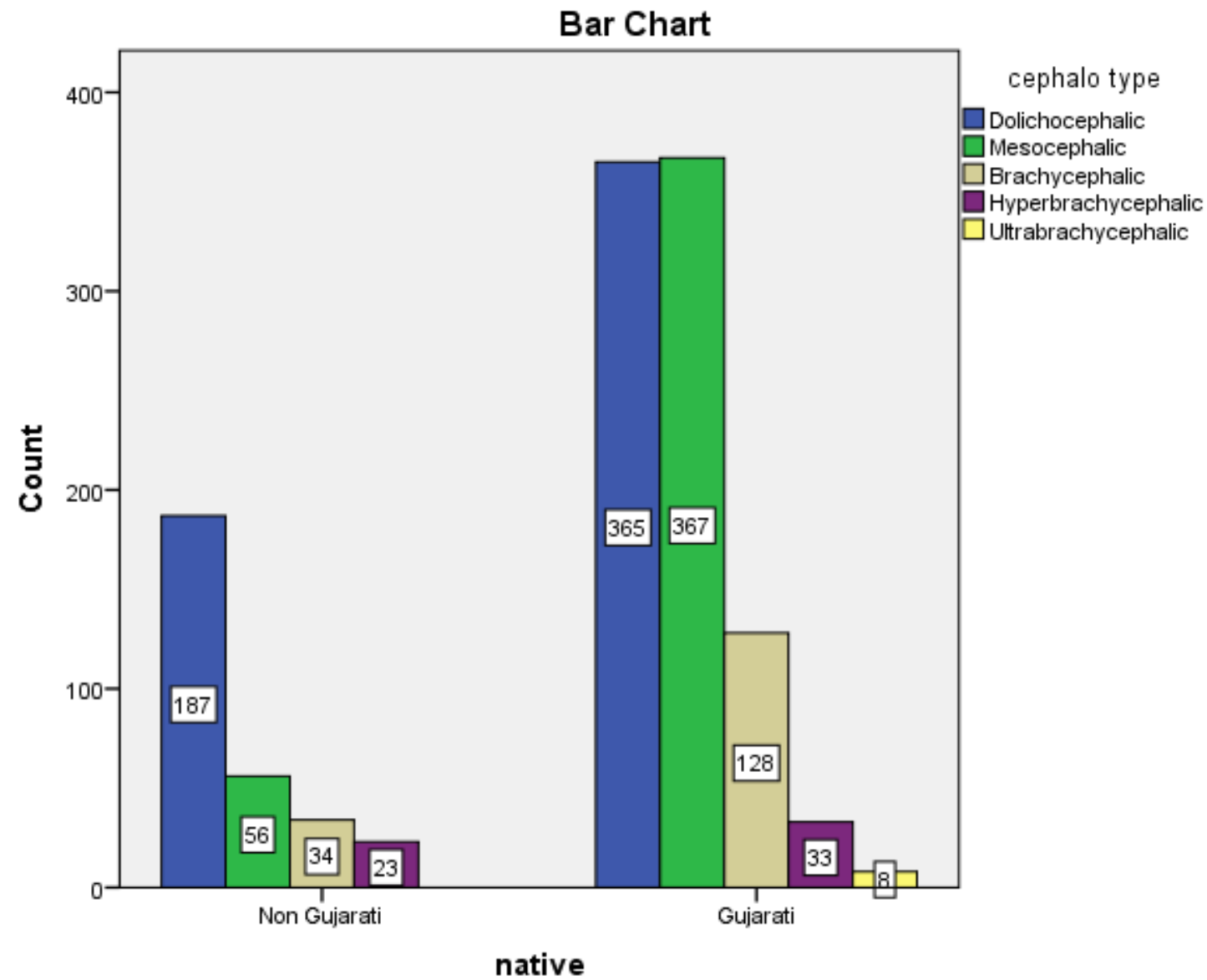

Figure 1: Cephalo type of Gujarati (901) and Non-Gujarati $(\mathrm{n}=300)$ 


\section{Bar Chart}

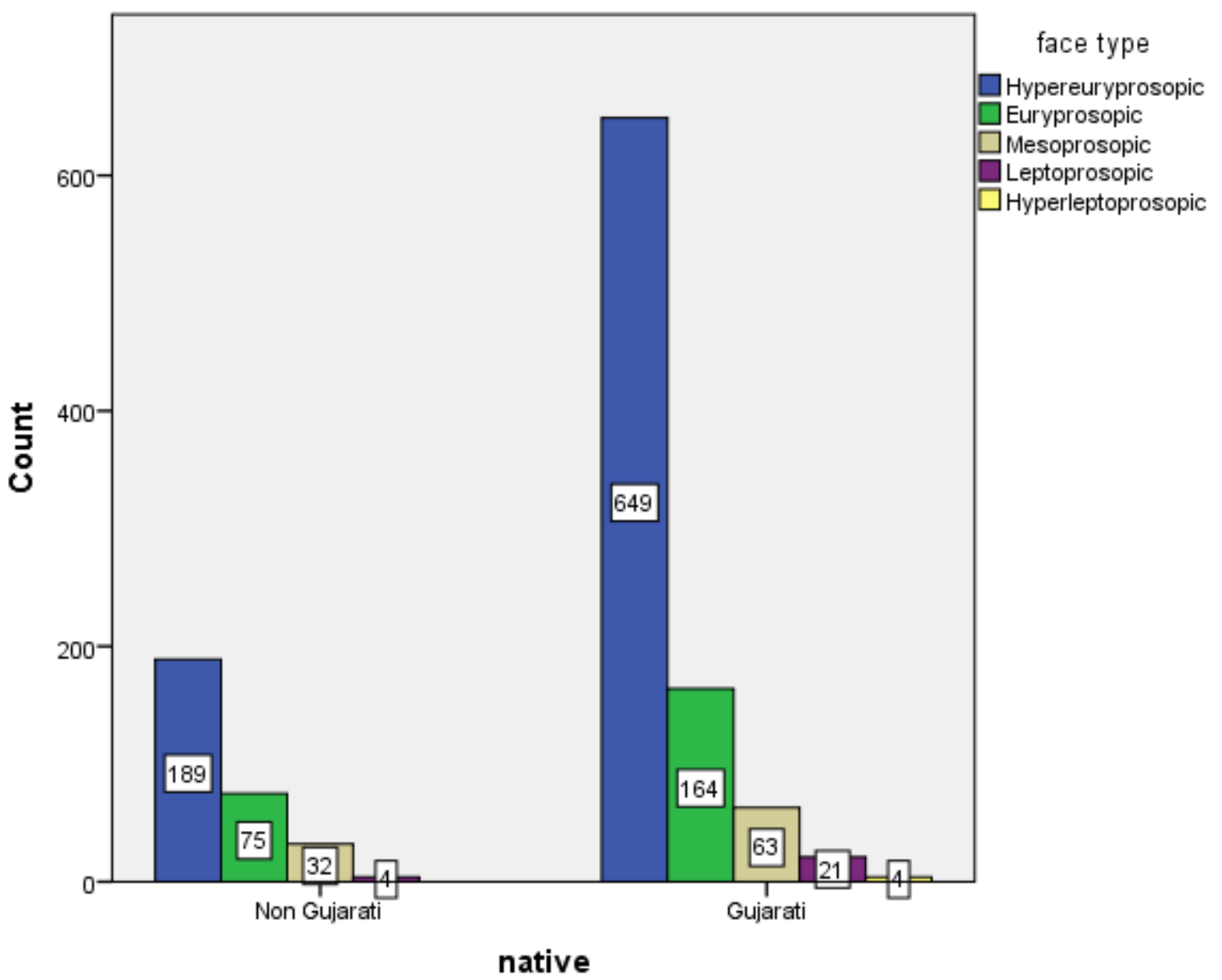

Figure 2: Facial type of Gujarati (901) and Non-Gujarati $(n=300)$

\section{Discussion}

Sexual dimorphism is an important concern for the forensic anthropologists as it is a key to individual identification. Assessing sexual dimorphism eliminates approximately half of the population from further considerations in cases of missing persons or unknown identity. Many morphological differences are sex specific. Recorded mean values for the measured variables, which determine the cephalofacial morphological features of Gujarati, in treated female entities (cephalic index $=77.38$, facial index $=$ 74.73 ) were comparatively lesser with respect to mean values in male entities (cephalic index $=77.149$, facial index $=75.354$ ) and in Non-Gujarati, female mean values (cephalic index $=81.170$, facial index $=76.952$ ) were greater in relation to the mean values of these variables in male entities (cephalic index $=70.615$, facial index $=74.283$ ). Hypothesis $1\left(\mathrm{H}_{\mathrm{o}}\right)$ states if there any significance difference in the mean values of all the variables in male and female of Gujarati and Non-Gujarati. According to the observations, it can be concluded that hypothesis $1\left(\mathrm{H}_{\mathrm{o}}\right)$ is accepted and there was a significant difference in the mean values of all the variables in male and female of Gujarati and Non-Gujarati which is important for sexual dimorphism. It can easily be made known from the above Table 4 that all the mean values of the measured variables were higher in Gujarati than Non- Gujarati group (Table 4). This is being important in determination of race. No statistical significant difference was seen in cephalic and facial index between Gujarati male and female as p-value $>0.05$ while other test variables showed positive significant difference ( $\mathrm{p}$-value $<0.05$ ). Consequently, Gujarati male and female can clearly be distinguished from the findings of length and width of their head and face. Non-Gujarati subject results show highly significant difference between sex (male and female) and test variables (head length, head width, facial length, facial width, cephalic index and facial index) which can be akey to sexual dimorphism (Table 3).

It was observed that the maximum head length ( $\mathrm{p}$-value $>0.05$ ) and facial index ( $\mathrm{p}$-value $>0.05$ ) were not found to have any significant relation with the studied group. The cephalic indices of Gujarati samples were $77.207 \pm 0.177$ which is greater in comparison to the average Non-Gujarati index $74.133 \pm 0.4242$. Table 5 depicts strong statistical significant difference $(\mathrm{p}-\mathrm{value}<0.05)$ in the measurements of head width, facial width, facial length and cephalic index in both the treated group. Hypothesis 2 tests the significance difference in the mean values of all variables for Gujarati and Non-Gujarati (Table 5). But hypothesis $2\left(\mathrm{H}_{\mathrm{o}}\right)$ may not be accepted in these cases as the mean cephalic and facial index in two groups varies significantly depending on genetic factor, nutritional growth and habitat. This differences leads to ethnic determination. 
It was concluded that dominant head types in Gujarati males was Mesocephalic (40.2\%) followed by Dolicocephalic (39.8\%). In females the dominant head types was Dolicocephalic (42.7\%) followed by Mesocephalic (42.2\%). According to the classification given by Martin and Saller (Table 1 and 2) (1957) [8], Gujarati male have medium head shape and female have long and narrow head. It was known that Non-Gujarati head types in male were long and narrow and in female were broad and short. Referring to the further classification, facial form of Gujarati and Non-Gujarati was known to be comparatively broad and short. According to Stewart's classification (1935) Gujarati subjects can be called mesati-cephalic. In the present study, the mean cephalic index of Gujarati was 77.2, whereas Dr. H.R.Jadav et al. [2], who studied on cephalic index of 180 males, was 80.20. Shah GV et al. [3] worked for cephalic index on 500 (302 male, 198 female) medical students of Gujarat aging 17-23 years only and concluded them to be brachycephalic with 80.81 index. Kanan Uttekar et al. [4] studied 800 subjects across various region of South Gujarat aging 18-25 years with mean cephalic index of 81.2 which is quiet high compare to our mean. In fact, our results are similar to Stewart's classification (1935) of Gujarati subjects belonging to mesocephalic head types.

In Gujarati the mean Facial index was 75.3 in males \& 74.7 in females. The mean facial index was 75.1. Our results showed that the dominant face type among Gujarati males were Hypereuryprosopic (76\%) followed by Euryprosopic (16.4\%) and in females were also Hypereuryprosopic (60\%), Euryprosopic (23.6\%). Hence it can be concluded that in Gujarati dominant face type was Hypereuryprosopic. Another study by Kanan Uttekar et al. [6] on facial index of adult males between 18-25 years age group residing in Gujarat states that the dominant face type was euryprosopic $42.96 \%$ followed by hypereuryprosopic $35.10 \%$ referring Banister classification [7]. Since he studied only males of younger age group therefore our results might vary with $76 \%$ of Gujarati with hypereuryprosopic face type.

These mean cephalic differences can be due to the sample size and age range selected in the respective works. As per the present study the mean cephalic index of Non-Gujarati in both the sexes was ranging from 70.6-81.1 and the mean cephalic index was 74.13. According to the percentage (\%), Non-Gujarati male entities were characterized with Dolicocephalic (80\%) followed by Mesocephalic (18\%) head types. Among Non-Gujarati females the principal head types were Brachycephalic (30\%), Dolicocephalic (27\%), Hyperbrachycephalic (23\%) and Mesocephalic (20\%). In Non-Gujarati, the most prominent face shape in males could be classified as Hypereuryprosopic with $76 \%$ and females are classified under Euryprosopic with $45 \%$. Leptoprosopic with $2 \%$ and Mesoprosopic with $18 \%$ was least common type in males and females respectively (Table 6 and 7). Also, Non-Gujarati subjects include people from various ethnic and racial backgrounds; it may be possible that male have lower cephalic and facial dimensions and indices than their females. Also, while collecting the samples the age groups of male were comparatively smaller than their female. Hence this can be the reason for contradict results of all previous reports in literature.

Further consideration was made and the studied data was cross tab for checking the most probable head and face form found in the population (Table 8 and 9). On analyzing, it was identified that when the head was recognized to be Mesocephalic and Dolicocephalic, the facial shape was predominantly be Hypereuryprosopic. Also when the head shape was known to be Brachycephalic, facial form was chiefly Euryprosopic in nature. Hence, from the above observations we can predict the dominant facial type among the study sample was Hypereuryprosopic.

Many researchers have worked on various ethnic group to find cephalic and facial indices and to know different head and face types commonly found in the referred community (Table 10). The findings are listed in Table 10.

\begin{tabular}{|c|c|c|c|}
\hline Authors & Work & Ethnic Group & Observations \\
\hline $\begin{array}{l}\text { Mahesh } \\
\text { Kumar [10] }\end{array}$ & $\begin{array}{l}\text { Study of Facial } \\
\text { Index }\end{array}$ & 600 Haryanvi adults & $\begin{array}{l}\checkmark \quad \text { Mean Facial Index Male }=86.09 \text { Mesoprosopic } \\
(49.66 \%) \\
\checkmark \quad \text { Mean Facial Index Female }=84.84 \text { Mesoprosopic }(35 \%)\end{array}$ \\
\hline $\begin{array}{l}\text { Zohre abatabaei } \\
\text { [11] }\end{array}$ & $\begin{array}{l}\text { Study of Facial } \\
\text { Index }\end{array}$ & 130 Patient of Yazd & $\begin{array}{ll}\checkmark & \text { Mean Facial Index Male }=108.39 \\
\checkmark & \text { Mean Facial Index Female }=106.98 \\
\checkmark & \text { No statistical difference between both sexes }\end{array}$ \\
\hline $\begin{array}{l}\text { Agron Rexhepi } \\
\text { [12] }\end{array}$ & $\begin{array}{l}\text { Cephalofacial } \\
\text { characteristics }\end{array}$ & $\begin{array}{l}754 \text { Kosova } \\
\text { subjects }\end{array}$ & $\begin{array}{ll}\checkmark & \text { Mean Cephalic Index Male }=83.59 \\
\checkmark & \text { Mean Cephalic Index Female }=84.79 \\
\checkmark & \text { Brachycephalic } \\
\checkmark & \text { Mean Facial Index Male }=90.38 \text { Leptoprosopic 31.2\% } \\
\text { and Hyperleptoprosopic } 31.6 \% \\
\checkmark \quad \text { Mean Facial Index Female }=90.27\end{array}$ \\
\hline $\begin{array}{l}\text { Vaishali R. Shetti } \\
\text { [13] }\end{array}$ & Facial Index & $\begin{array}{l}300 \text { Indian and } \\
\text { Malaysian students }\end{array}$ & $\begin{array}{l}\checkmark \quad \text { Mean Facial Index Malaysian Male }=85.72 \text { Eurypros- } \\
\text { opic } \\
\checkmark \quad \text { Mean Facial IndexMalaysian Female }=87.71 \text { Meso- } \\
\text { prosopic } \\
\checkmark \quad \text { Mean Facial Index Indian Male }=87.19 \text { Mesoprosopic } \\
\checkmark \quad \text { Mean Facial Index Indian Female }=86.75 \text { Mesopros- } \\
\text { opic and Euryprosopic }\end{array}$ \\
\hline
\end{tabular}




\begin{tabular}{|c|c|c|c|}
\hline Authors & Work & Ethnic Group & Observations \\
\hline H.A.Alvies [14] & Study of Cephalic Index & $\begin{array}{l}110 \text { Male students } \\
\text { of North and South } \\
\text { Brazil }\end{array}$ & $\begin{array}{lll}\checkmark & \text { Mean Cephalic } & \text { Index North Brazil }=80.93 \\
\checkmark & \text { Mean Cephalic } & \text { Index South Brazil }=79.06 \\
\checkmark & \text { Brachycephalic } & \end{array}$ \\
\hline $\begin{array}{l}\text { Dr. H.R.Jadav } \\
{[2]}\end{array}$ & Cephalic Index & $\begin{array}{l}180 \text { subjects of Sindhi, } \\
\text { Patel, Rabari, Kharwa, } \\
\text { Bhil, Siddi of Gujarat }\end{array}$ & $\checkmark \quad$ Mean Cephalic Index $=80.20$ \\
\hline $\begin{array}{l}\text { Praveen Kumar } \\
{[15]}\end{array}$ & $\begin{array}{l}\text { Cephalic and } \\
\text { Facial Index }\end{array}$ & $\begin{array}{l}100 \text { Male South } \\
\text { Indian subjects }\end{array}$ & $\begin{array}{ll}\checkmark & \text { Mean Cephalic Index }=76.78 \\
\checkmark & \text { Mean Facial Index }=90.95\end{array}$ \\
\hline $\begin{array}{l}\text { V. Raveendra } \\
\text { Nath [16] }\end{array}$ & $\begin{array}{l}\text { Cephalic Index, Cranial } \\
\text { Volume and Cranial meas- } \\
\text { urements in cadavers }\end{array}$ & $\begin{array}{l}93 \text { formalin cadavers } \\
\text { South Indian origin }\end{array}$ & $\begin{array}{ll}\checkmark & \text { Mean Cephalic Index }=77.98 \text { Mesocephalic } \\
\checkmark & \text { Mean Cephalic Index Male }=76.97 \\
\checkmark & \text { Mean Cephalic Index Female }=79.2\end{array}$ \\
\hline $\begin{array}{l}\text { Isurani } \\
\text { Ilayperuma [17] }\end{array}$ & Cephalic Indices & $\begin{array}{l}400 \text { Sri Lankan } \\
\text { subjects }\end{array}$ & $\begin{array}{ll}\checkmark & \text { Mean Cephalic index }=78.54 \\
\checkmark & \text { Brachycephalic }\end{array}$ \\
\hline $\begin{array}{l}\text { Shah G.V. } \\
\text { Jadav [3] }\end{array}$ & Cephalic Index & $\begin{array}{l}500 \text { Gujarati } \\
\text { subjects }\end{array}$ & $\begin{array}{ll}\checkmark & \text { Mean Cephalic Index }=80.81 \\
\checkmark & \text { Brachycephalic } \\
\checkmark & \text { Mean Cephalic Index Male }=80.42 \\
\checkmark & \text { Mean Cephalic Index Female }=81.0\end{array}$ \\
\hline $\begin{array}{l}\text { Swapnali } \\
\text { Khair [18] }\end{array}$ & Cephalic Index & 100 Mumbai subjects & $\begin{array}{ll}\checkmark & \text { Mean Cephalic Index }=78.48 \\
\checkmark & \text { Mesocephalic } \\
\checkmark & \text { Mean Cephalic Index Male }=81.28 \\
\checkmark & \text { Brachycephalic } \\
\checkmark & \text { Mean Cephalic Index Female }=75.22 \\
\checkmark & \text { Mesocephalic }\end{array}$ \\
\hline $\begin{array}{l}\text { Dr. Sunita } \\
\text { Patro [18] }\end{array}$ & Cephalic Index & $\begin{array}{l}1030 \text { Odisha } \\
\text { patients }\end{array}$ & $\begin{array}{ll}\checkmark & \text { Mean Cephalic Index }=77.75 \\
\checkmark & \text { Mesocephalic } \\
\checkmark & \text { Mean Cephalic Index Male }=77.28 \\
\checkmark & \text { Mean Cephalic Index Female }=78.38\end{array}$ \\
\hline $\begin{array}{l}\text { Vishal } \\
\text { Manoharrao } \\
\text { Salve [20] }\end{array}$ & Cephalic Index & $\begin{array}{l}300 \text { Andhra region } \\
\text { adults }\end{array}$ & $\begin{array}{ll}\checkmark & \text { Mean Cephalic Index }=76.94 \\
\checkmark & \text { Mean Cephalic Index Male }=75.68 \text { Dolicocephalic } \\
\checkmark & \text { Mean Cephalic Index Female }=78.20 \\
\checkmark & \text { Mesocephalic }\end{array}$ \\
\hline $\begin{array}{l}\text { Dr. Anupama } \\
\text { Mahajan [21] }\end{array}$ & Cephalic Index & $\begin{array}{l}400 \text { Punjabi medical } \\
\text { students }\end{array}$ & $\begin{array}{ll}\checkmark & \text { Mean Cephalic Index }=85.53 \\
\checkmark & \text { Brachycephalic and Hyperbrachycephalic } \\
\checkmark & \text { Mean Cephalic Index Male }=81.64 \\
\checkmark & \text { Mean Cephalic Index Female }=85.75\end{array}$ \\
\hline $\begin{array}{l}\text { Kanan } \\
\text { Uttekar [4] }\end{array}$ & Cephalic index & 800 Gujarati & $\begin{array}{ll}\checkmark & \text { Mean Cephalic Index }=81.2 \\
\checkmark & \text { Mean Cephalic Index Male }=80.88 \\
\checkmark & \text { Mean Cephalic Index Female }=82.48\end{array}$ \\
\hline $\begin{array}{l}\text { Vaishali } \\
\text { Yagain [22 }\end{array}$ & Cephalic Index & 100 Indian students & $\begin{array}{ll}\checkmark & \text { Mean Cephalic Index }=79.38 \\
\checkmark & \text { Mean Cephalic Index Male }=77.92 \\
\checkmark & \text { Mesocephalic } \\
\checkmark & \text { Mean Cephalic Index Female }=80.85 \\
\checkmark & \text { Brachycephalic }\end{array}$ \\
\hline Lobo SW [23] & Cephalic Index & $\begin{array}{l}267 \text { Gurung } \\
\text { community, Nepal }\end{array}$ & $\begin{array}{ll}\checkmark & \text { Mean Cephalic Index }=83.7 \\
\checkmark & \text { Mean Cephalic Index Male }=83.1 \\
\checkmark & \text { Mean Cephalic Index Female }=84.6 \\
\checkmark & \text { Brachycephalic }\end{array}$ \\
\hline Present Work & $\begin{array}{l}\text { Cephalic and } \\
\text { Facial Index }\end{array}$ & $\begin{array}{l}901 \text { Gujarati and } \\
\text { 300Non-Gujarati } \\
\text { adults, India }\end{array}$ & $\begin{array}{ll}\checkmark & \text { Mean Cephalic Index of Gujarati }=77.2 \\
\checkmark & \text { Mean Facial Index of Gujarati }=75 \\
\checkmark & \text { Mean Cephalic Index of Non- Gujarati }=75.8 \\
\checkmark & \text { Mean Facial Index of Non- Gujarati }=75.5\end{array}$ \\
\hline
\end{tabular}

Table 10: Various Study on Cephalic and facial indices

With the help of the above statistics, the sex and ethnicity can be known with the cephalofacial measurements and indices. This knowledge can be of immense importance to anthropologists as well as forensic science experts as a means of identification.

\section{Conclusion}

From the results of the present study the two treated groups showed statistically significant differences in mean values of all the measured cephalofacial variables namely head height, head width, facial height and facial width which successfully predict anthropometric relationships between two population groups. The significant difference between male and female was observed in head and face measurements of Gujarati. The study showed predominantly mesocephalic, dolicocephalic and hypereuryprosopic 
head and face forms among Gujarati and dolicocephalic, brachycephalic, hypereuryprosopic and euryprosopic types among NonGujarati. Therefore sexual dimorphism and ancestral difference is expressed from the study. The present study provides new and valuable data pertaining to cephalic and facial indices and the shapes of the head and face in Gujarati and Non-Gujarati individuals between 20-50 years of age, belonging to Ahmedabad district of Gujarat. This result is of great importance in medico- legal and forensic science as marker of ethnicity and sex.

\section{Acknowledgement}

One of the authors, Twisha Shah gratefully acknowledges University Grant Commission (UGC), New Delhi for financial support.

\section{References}

1. Goldstein MS (1936) Changes in dimensions and form of the face and head with age. Am J Phys Anthropol 22: 37-89.

2. HR Jadav, VB Kariya, BB Kodiyatar, CA Pensi (2011) A Study to Correlate Cephalic Index of Various Caste/Races Of Gujarat State. NJIRM 2: 18-22.

3. Shah GV, Jadhav HR (2004) The Study of Cephalic index in Students of Gujarat. J Anat Soc India 53: 25-6.

4. Kanan U, Gupta DS, Andani R, Kubavat DM, Nagar, et al. (2013) Study of Cephalic Index in South Gujarat. Int J Recent Trends in Sci Technol 8: 87-9.

5. Mehta M, Saini V, Nath S, Patel MN, Menon SK (2014) CT scan images to determine the origin from craniofacial indices for Gujarati population. Journal of Forensic Radiol Imag 2: 64-71.

6. Kanan U, Gandotra A, Desai A, Andani R (2012) Variation inFacial index of Gujarati Males - A Photometric study. Int J Med Health Sci 1: 64-5

7. Williams P, M Dyson, JE Dussak, LH Bannister, MM Berry, et al. (1995) Gray’s Anatomy. In: Skeletal system. 38th Ed. Elbs with Churchill Livingston. London. 607-12.

8. Martin R, K Saller (1957) Lehrbuch der anthropologie. Gustav Fischer Verlag, Stuttgart.

9. Singh IP, Bhasin MK (1989) A laboratory manual on biological anthropology. $2^{\text {nd }}$ rev ed, Delhi: Nazia Offset Press.

10. Kumar M, Lone MM (2013) The Study of Facial Index among Haryanvi Adults. IJSR 2: 51-3.

11. Tabatabaei Z, Yasaei S, Ardakani MD, Said AM (2010) Assignment and compression of facial index and modified smile index (MSI). Iranian Journal of Orthodontics 5: 70-6.

12. Rexhepi A, Meka V (2008) Cephalofacial Morphological Characteristics of Albanian Kosova Population. Int J Morphol 26: 935-40.

13. Shetti VR, Pai SR, Sneha GK, Gupta C, Chethan P, et al. (2011) Study of Prosopic (Facial) Index of Indian and Malaysian Students. Int J Morphol 29: 1018-21.

14. Alves AH, Santos MIMP, Melo FCL, Wellington R (2011) Comparative Study of the Cephalic Index of the Population from the Regions of the North and South of Brazil. Int J Morphol 29: 1370-74.

15. Doni PKR, Janaki CS, Vijayaraghavan V, Delhi Raj U (2013) A Study on Measurement and Correlation of Cephalic and Facial Indices in Males of South Indian Population. Int J Med Res. 2: 439-46.

16. V. Raveendranath, K. Y. Manjunath. An Anthropometric Study of Correlation between Cephalic Index, Cranial Volume and Cranial Measurements in Indian Cadavers.

17. Ilayperuma I (2011) Evaluation of Cephalic Indices: A Clue for Racial and Sex Diversity. Int J Morphol 29: 112-7.

18. Khair S, Bhandari D, Wavhal S (2013) Study of Cephalic Index among the Students of Mumbai Region. Indian J Applied Res. 3: 64-6.

19. Patro S, Sahu R, Rath S (2014) Study of cephalic index in Southern Odisha Population. J Dent Med Sci 13: 41-4.

20. Salve VM, Thota NR, Patibandla A (2011) The Study of Cephalic Index of Andhra Region (India). Asian J Med Sci 2: 53-5.

21. Mahajan A, Khurana BS, Seema, Batra APS (2009) The Study of Cephalic Index in Punjabi Students. J Punjab Acad Forensic Med Toxicol 9: 66-70.

22. Yagain VK, Pai SR, Kalthur SG, Chethan P, Hemalatha I (2012) Study Of Cephalic Index In Indian Students. Int J Morphol 30: $125-9$.

23. Lobo SW, Chandrashekhar TS, Kumar S (2005) Cephalic Index of Gurung Community of Nepal - An Anthropometric Study. Kathmandu Univ Med J 3: 263-5.

24. Stewart TD (1935) Anthropometric nomenclature II. The cephalic index, American J anthropology 97-140. 